\title{
ESTIMATED THRESHOLD VALUE OF INITIAL AORTIC STIFFNESS FOR ASSESSMENT OF THE EFFECT OF AEROBIC PHYSICAL TRAINING IN METABOLIC SYNDROME SUBJECTS
}

Dr. leva Slivovskaja ${ }^{1,3}$, Dr. Ligita Ryliskyte ${ }^{1,3}$, Jurate Balsyte ${ }^{1,2}$, Jurgita Buzinskaite ${ }^{3}$ (1) Medical Faculty of Vilnius University; (2) State Research Institute for Innovative Medicine; (3) Vilnius University Hospital Santaros Klinikos. Vilnius, Lithuania

\section{Introduction.}

Heart rate (HR) controlled aerobic physical training (APT) is well accepted for the physical training of subjects with metabolic syndrome (MetS). Aortic stiffness measured by carotid - femoral pulse wave velocity (cfPWV) is considered to be the significant surrogate quantitative marker of cardiovascular risk.

\section{Purpose.}

We aimed to assess the relationship between the cfPWV before and after ATP and to estimate the threshold value of initial aortic stiffness reflective the effect of training.

\section{Method.}

The study included 84 obese middle-aged (53,9 $\pm 6,4$ years old, 59,52\% women) subjects with MetS (updated NCEP ATP III) without overt cardiovascular disease. Subjects were randomly recruited for moderate intensity APT from the participants of the Lithuanian High Cardiovascular Risk (LitHiR) primary prevention programme. Individually by cardiorespiratory testing estimated heart rate was used as target for ATP. Aortic stiffness was assessed by measuring of cfPWV before and after 2 months of ATP. The ATP consisted of 8-week supervised aerobic training 30-40 min/day, 5 days/wk.

\section{Results.}

Statistically significant negative correlation between the cfPWV measured before and after APT was found. In case of higher initial cfPWF value more pronounced diminishing of cfPWV after APT took place. Coefficient of correlation was $-r=0656$ (moderate strength negative relationship, $\mathrm{p}<0.001)$.

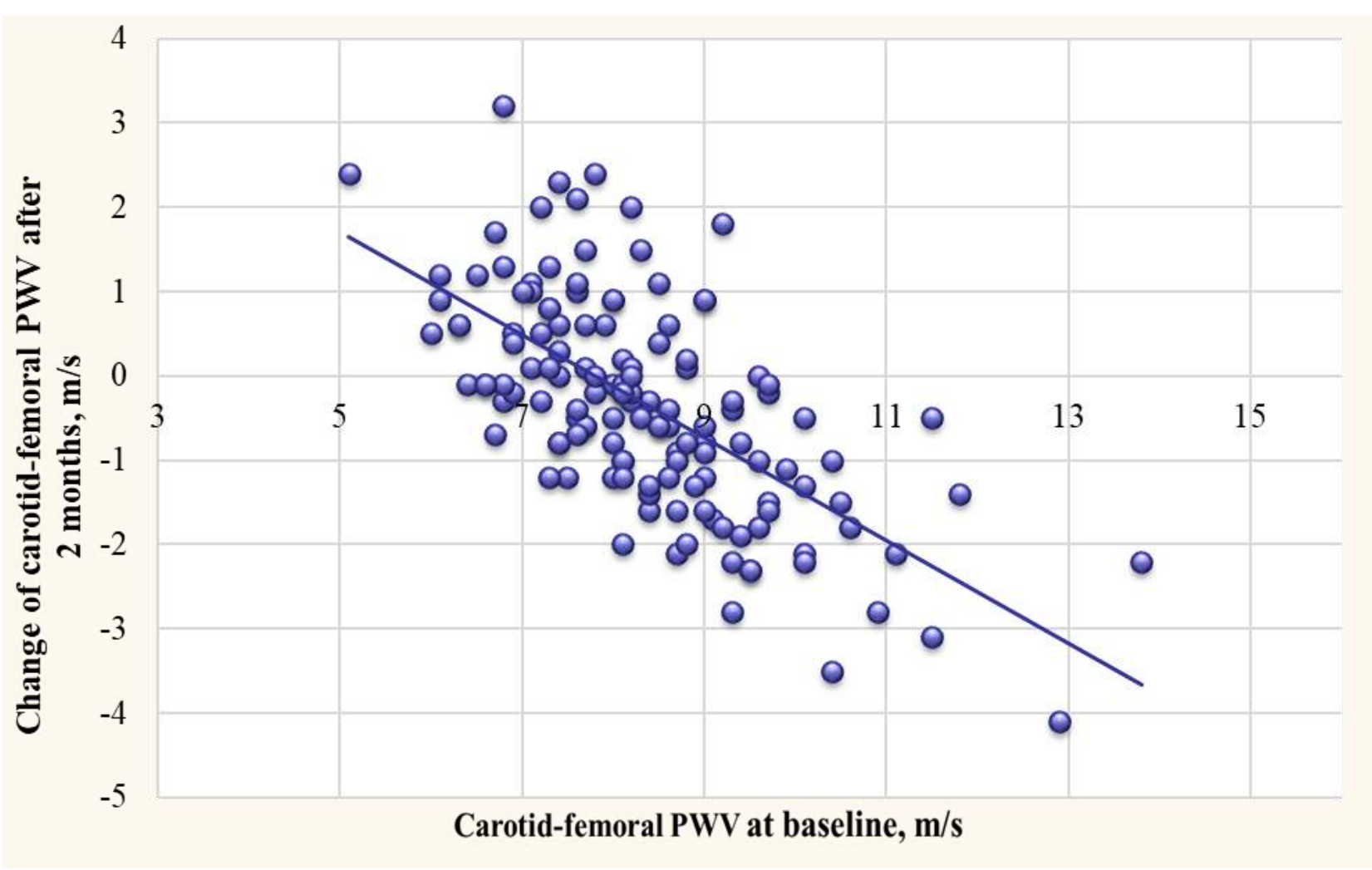

In order to estimate the threshold initial cfPWV value discriminating MetS subjects in whom aortic stiffness will improve after APT with maximal prognostic accuracy the ROC curve analysis was performed. The estimated discriminating threshold value of initial cfPWV was $8.1 \mathrm{~m} / \mathrm{s}$ (under the curve 0.8175).

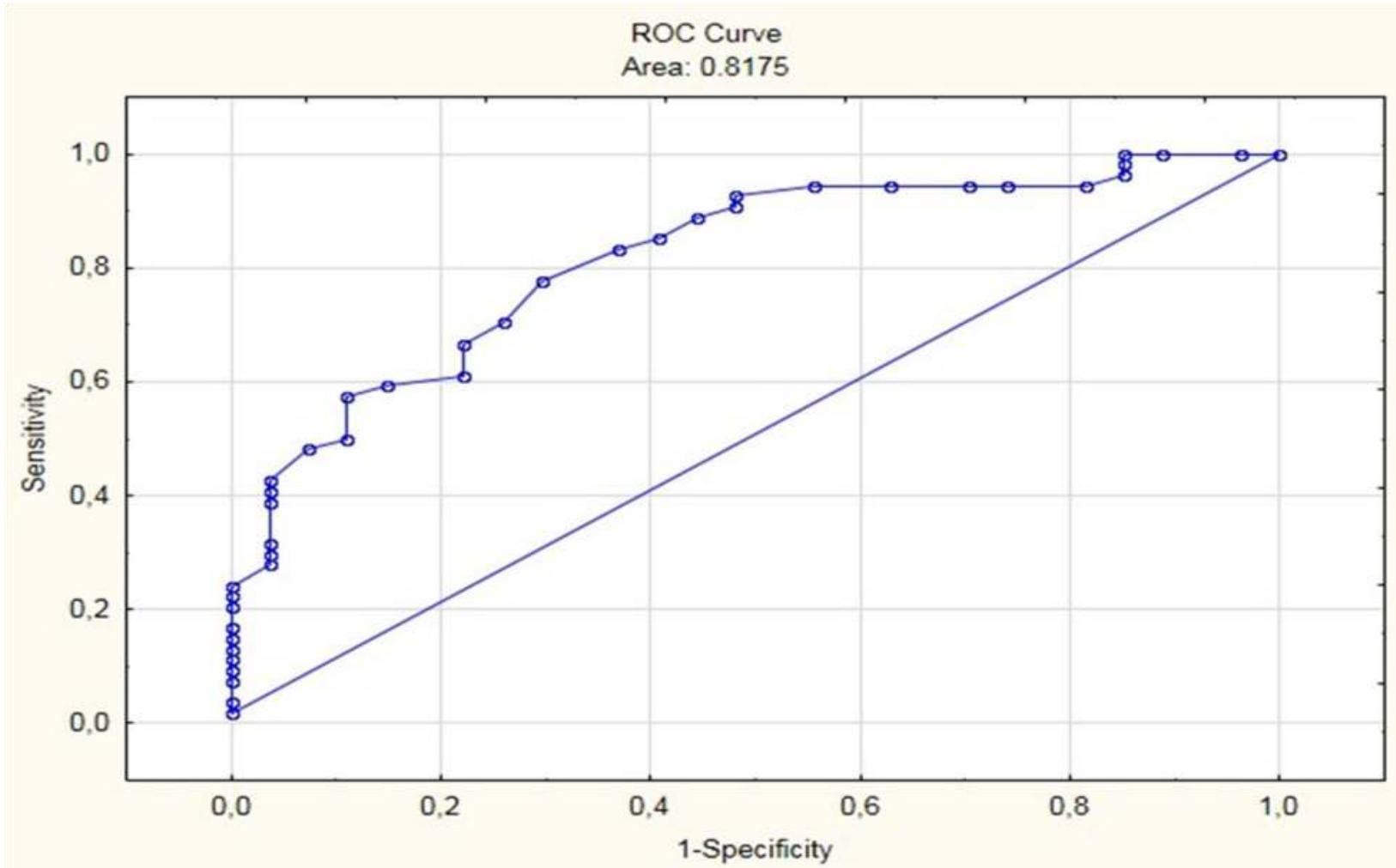

\section{Conclusions.}

Aerobic training programme significantly improved aortic stiffness when initial value of cfPWV was $>8.1 \mathrm{~m} / \mathrm{s}$.
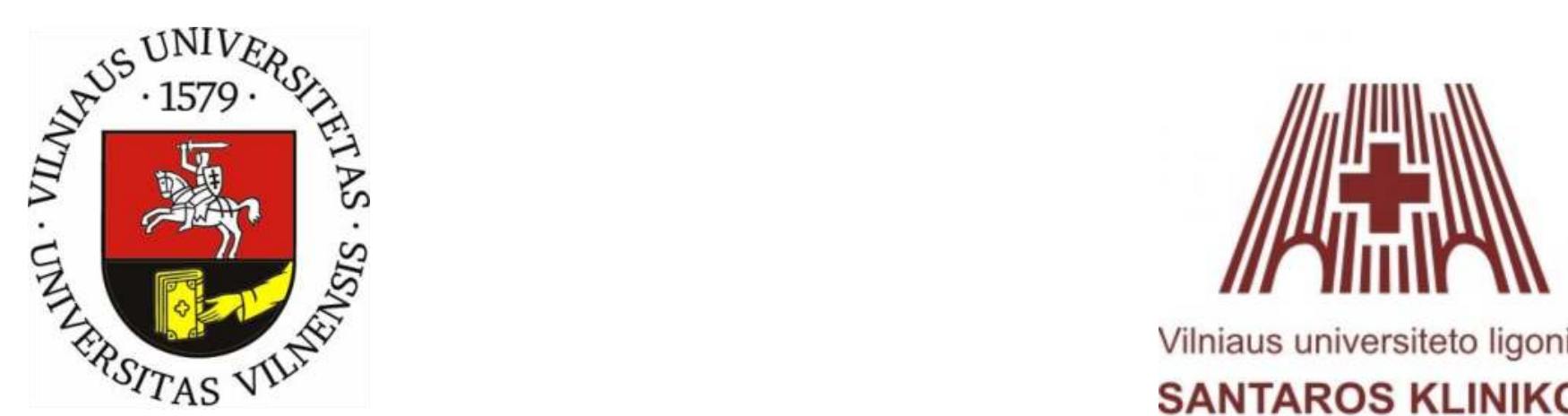